\title{
Role of Artificial Intelligence in Continuous Monitoring of Patients and Reducing Code Blue Events in a Tertiary Care Hospital - Our Experience
}

\author{
Anil Kumar T, Vishwanath K, Ashwin K*, Tharanath S and Sujatha \\ KJ \\ Ramaiah Medical College, Hospital Bangalore, India
}

*Corresponding author: Ashwin Kulkarni, Ramaiah Medical College, Hospital Bangalore, Flat No- A-109, Hoysala Commanders retreat, 6th cross, Bhadrappa Layout, Kodigehalli, Bangalore 560094, India, Tel: 9620186394; Email: drahwinkulkarni@yahoo.in

\section{Abstract}

Artificial Intelligence (AI) has brought lot of advancement in various fields. Even in health care areas AI has made lot of impact in improvising the medical care and management. Continuous monitoring of patients is a vital aspect in the management of patients. There are many instances where even stable patients suddenly deteriorate in the wards. Continuous monitoring is the required to avoid such catastrophic events. We have implemented Cloud connected continuous monitoring systems in acute medicine ward in Ramaiah Medical College Hospital. Acute Medicine ward is a high dependency ward where vulnerable patients who require continuous monitoring are admitted. We found that Cloud connected continuous monitoring systems was helpful in timely identification of the critical events, reducing code blue events and reduced burden on Intensive care units. Here in this article we present our experience by utilizing Cloud connected continuous monitoring systems at our hospital.

Keywords: Cloud connected continuous monitoring systems; Artificial Intelligence; Code Blue

\section{Introduction}

Artificial Intelligence (AI) has created a revolution in the modern era in various fields. Artificial Intelligence has brought lot of modern technologies in the management of various diseases in hospitals. Artificial Intelligence is now playing an active role in creating smart hospitals where AI helps the doctor and patients in the management of illness with ease and safety. We have implemented one such technology in the continuous monitoring system at our hospital. Continuous monitoring is the cornerstone in the management of patients in nonICU settings. Continuous monitoring systems have revolutionized management of vulnerable patients which alerts the doctors to identify the critical events and intervene timely. They have a significant role in non-ICU setting where they can be used to reduce the burden on ICU and also provide safety to the patients.

Continuous monitoring in the Non-ICU setting has been shown to reduce ICU readmissions and hospital lengths of stay. In fact, up to $75 \%$ of non-do-not- 


\section{Medical Journal of Clinical Trials \& Case Studies}

resuscitate (DNR) hospital deaths occur in unmonitored settings outside the ICU. Currently, monitoring outside the ICU is a manual process with nurses spending nearly $15 \%$ of their time collecting and documenting vitals. We implemented a continuous monitoring system in our acute medicine ward (High dependence ward) to manage vulnerable patients who required close surveillance. We found that the availability of the continuously monitored systems would improve the management of patients in wards, improve patient safety, and improve ICU efficiency making it available only for the highest risk patients requiring interventional care.

\section{Our Experience}

We implemented a Cloud-connected continuous monitoring system for monitoring vulnerable patients who get admitted to the wards in Ramaiah Medical College Hospital Bangalore. This group of patients are stable to be admitted to the wards but require continuous monitoring like geriatric patients, patients with multiple comorbidities, anemia, gastroenteritis with dehydration, Viral thrombocytopenic fevers. Continuous monitoring systems allow clinicians to monitor patients remotely on their smartphones. It measures key vital signs like heart rate, blood pressure, oxygen saturation, temperature, respiratory rate and ECG.

Key features are

1. Automates manual monitoring \& documentation of vitals increasing nursing productivity.

2. Provides patient vital sign information every 5 minutes to generate high-resolution long-term trends that ensure the patient safety.

3 . By remotely connecting patients to doctors through the smartphone, ensures proactive clinical decisionmaking to improve patient outcomes.

The continuous monitoring systems also provide the alerts to the clinician in case of a critical event. The continuous monitoring system through its in built artificial intelligence provides the clinician an alert that the patient is likely to deteriorate even 15 minutes prior to the critical event. We have implemented this system in our hospital since 18 months. Monitoring systems also alert the doctors using Emergency warning scores (EWS). These warning scores stratify the patients at high risk and low risk status. We also noted there was significant reduction in the code blue events in the wards. We had many instances where patient monitoring systems could identify life threatening critical event which was identified and treated timely.
1. A 70 year old gentleman was admitted to a high dependency ward for viral fever. At around 1 am he developed severe bradycardia (Heart rate of 40 beats /min). This was alerted to the doctor in charge of the patient by the monitor, which was then diagnosed as complete heart block and was managed according.

2. We had a patient admitted with Bronchopneumonia and Asthma. His vital parameters were stable throughout the admission. He suddenly developed breathlessness at $2 \mathrm{pm}$. This was identified by the continuous monitoring system. He had tachypnea, tachycardia and low oxygen saturation. This was immediately alerted to the duty doctor and was managed accordingly.

\section{Discussion}

We implemented continuous monitoring systems for vulnerable patients getting admitted to wards. Majority of our patients were febrile illness, anemias, Diabetics, Respiratory infections. In a study conducted by Barbara J. Drew et al. 18\% monitoring systems were used for treatment of cardiac medical or surgical diagnosis, $43 \%$ were treated for a neurologic or neurosurgical diagnosis, and 39\% were treated for another medical-surgical (pulmonary, sepsis, multi-system organ failure, etc) diagnosis. Cloud based continuous monitors could identify the critical events during the patient stay like arrhythmias, hypotension, desaturation [1]. Similarly according to the study conducted by Xiao Hu et al. cardiac and respiratory arrests accounted for $74 \%$ and $22 \%$ of cases, respectively [2]. It was observed that there was significant reduction of ICU load and many vulnerable patients could be monitored in Pre-ICU settings. This was in accordance with a study conducted by Taenzer, et al. which showed a statistically significant reduction of $2.1 \%$ in ICU admissions post the implementation of continuous monitoring systems. A study conducted by Eyal Zimlichman, et al. also showed a $39.5 \%$ decrease in ICU transfers [3]. On the contrary, a study conducted by Brown, et al. the overall ICU admission rates did not change even after implementation of the monitoring systems [4]. With the implementation of continuous monitoring systems, there was a significant reduction in the number of patients admitted to the ICU as they were managed in the Acute Medicine Ward [5,6]. Prior studies in this area have focused on vital signs at the time of admission.

In this study we were able to validate the Modified Early Warning Score (MEWS) as good predictors of 


\section{Medical Journal of Clinical Trials \& Case Studies}

mortality at any time point during the period of hospitalization. There was significant reduction in the code blue events. This was as opposed to a study conducted by Xiao $\mathrm{Hu}$, et al. where among the patients monitored by alarm systems, majority of code blue events occurred in ICUs (68\%) with $23 \%$ code blue events occurring in non-ICU units, and $9 \%$ in other facilities including $\mathrm{OR}$ and interventional suits. The overall inhospital mortality for the cohort in our study was nil as opposed to the study by Barbara J. Drew, et al. where the mortality rate of patients being monitored using continuous monitoring systems was $7.6 \%[1,2]$.

\section{Conclusions}

Cloud Connected continuous monitoring of vulnerable patients is safe and efficacious. It reduced the burden on ICU wherein ICU beds can be utilized for more sick patients who need more intensive management. It reduces the incidence of code blue events in the wards. Critical worsening parameters can be identified at the right time and interventions can be done.

1. Continuous monitoring in non-ICU setting reduced patient burden on ICU and ensured use of ICU beds for the most deserving cases.

2. Continuous monitoring systems increased patient safety and reduced code blue rates in the wards.

3. Continuous monitoring reduced clinical burden due to availability of remote monitoring capabilities.

4. Continuous monitoring substantially reduced medical expenses for the patients with improved clinical outcomes.

5. We recommend continuous monitoring for the following subset of patients to improve outcomes, and operational efficiencies.

a. High-risk pregnancies and complicated deliveries for at least 24 hours post-delivery.

b. Post-operative cases and major surgical cases for at least 24 hours post-surgery.

c. Geriatric patients with co-morbidities for the first 48 hours after admission. d. COPD patients with requirement of domiciliary oxygen therapy.

e. Patients moved from the ICU for the first 24 hours.

f. Post-transplant patients to ensure early detection of deterioration real world settings and extension to mobile computing.

\section{References}

1. Drew BJ, Harris P, Ze`gre-Hemsey JK, Tina Mammone, Daniel Schindler, et al. (2014) Insights into the Problem of Alarm Fatigue with Physiologic Monitor Devices: A Comprehensive Observational Study of Consecutive Intensive Care Unit Patients. PLOS one 9(10): e110274.

2. Hu X, Sapo M, Nenov V, Barry T, Kim S, et al. (2012) Predictive combinations of monitor alarms preceding in-hospital code blue events. J Biomed Inform 45(5): 913-921.

3. Taenzer AH, Pyke JB, McGrath SP, Blike GT (2010) Impact of pulse oximetry surveillance on rescue events and intensive care unit transfers: a beforeand-after concurrence study. Anesthesiology 112(2): 282-287.

4. Zimlichman E, Terrence J, Argaman D, Shinar Z, Brown H (2012) Effect of contactless continuous patient monitoring in a medical-surgical unit on intensive care unit transfers: A Controlled Clinical Trial. ATS journals 185: 1-A3793.

5. Brown H, Terrence J, Vasquez P, Bates DW, Zimlichman E (2014) Continuous monitoring in an inpatient medical-surgical unit: A controlled clinical trial. Am J Med 115(2): 421-431.

6. Loon K, Zaane B, Bosch EJ, Cor J Kalkman, Linda M Peelen (2015) Non-Invasive Continuous Respiratory Monitoring on General Hospital Wards: A Systematic Review. PLOS One 10(12): e0144626. 